HRI-P-09-09-002

RECAPP-HRI-2009-018

CU-PHYSICS/06-2009

\title{
Search for Higgs bosons of the Universal Extra Dimensions at the Large Hadron Collider
}

\author{
Priyotosh Bandyopadhyay ${ }^{a}$, Biplob Bhattacherjee 2 , AseshKrishna Datta 4 \\ ${ }^{a}$ Regional Centre for Accelerator-based Particle Physics (RECAPP) \\ Harish-Chandra Research Institute 4 \\ Chhatnag Road, Jhunsi, Allahabad, India 211019 \\ ${ }^{b}$ Department of Physics \\ University of Calcutta, 92 A.P.C. Road, Kolkata, India 700009
}

\begin{abstract}
The Higgs sector of the Universal Extra Dimensions (UED) has a rather involved setup. With one extra space dimension, the main ingredients to the construct are the higher Kaluza-Klein (KK) excitations of the Standard Model Higgs boson and the fifth components of the gauge fields which on compactification appear as scalar degrees of freedom and can mix with the former thus leading to physical KK-Higgs states of the scenario. In this work, we explore in detail the phenomenology of such a Higgs sector of the UED with the Large Hadron Collider (LHC) in focus. We work out relevant decay branching fractions involving the KK-Higgs excitations. Possible production modes of the KK-Higgs bosons are then discussed with an emphasis on their associated production with the third generation KK-quarks and that under the cascade decays of strongly interacting UED excitations which turn out to be the only phenomenologically significant modes. It is pointed out that the collider searches of such Higgs bosons face generic hardship due to soft end-products which result from severe degeneracies in the masses of the involved excitations in the minimal version of the UED (MUED). Generic implications of either observing some or all of the KK-Higgs bosons at the LHC are discussed.
\end{abstract}

\footnotetext{
${ }^{1}$ priyotosh@hri.res.in

${ }^{2}$ biplob@gmail.com

${ }^{3}$ asesh@hri.res.in

${ }^{4}$ A constituent institute of Homi Bhabha National Institute (HBNI), Department of Atomic Energy, Government of India
} 


\section{Introduction}

In recent times, the Universal Extra Dimensions (UED) proposed by Applequist, Cheng and Dobrescu (the ACD framework) [1] has emerged as a viable option for physics beyond the Standard Model (SM) (see also [2] for earlier related ideas). In the UED, the extra space dimensions (the bulk) are available universally to all the SM particles to propagate in, viz., the fermions, the gauge bosons and the only scalar (the Higgs boson) of the SM. The simplest incarnation of such a scenario has only one extra space dimension (i.e., a $4+1$ dimensional space-time) and is popularly known in the literature as the minimal UED (MUED) framework. With the Large Hadron Collider about to begin its operation, the scenario attracted a lot of attention in terms of its discovery and distinction from an extremely popular alternative in the form of supersymmetry (SUSY) which could potentially fake the observations.

It is well-known that in a theory with odd number of space-time dimensions (like the case of MUED with 5 space-time dimensions), one cannot have chiral fermions, which is an observational fact. Compactification of the extra space dimension on an orbifold $\left(S^{1} / Z_{2}\right.$, in the present case) results in chiral fermions in the effective 4-dimensional theory [1]. Also, only four of the five components of the gauge bosons survive in the low energy theory. Thus, orbifold compactifications opens up the avenue of identifying the $\mathrm{SM}$ as the low-energy limit of a $\mathrm{TeV}$-scale extra dimensional theory.

Compactification of the extra space dimension leads to infinite towers of excitations (the Kaluza-Klein (KK) excitations) in the effective 4-D theory for the fields which originally have access to it (the bulk). By construct, thus, all the SM particles (quarks, leptons, gauge-bosons and Higgs) have their KK-excitations in the effective 4-D theory. Also, in the process, other towers of KK-excitations emerge which do not have their SM counterparts. The presence of KK-towers for charged and neutral CP-odd Higgs bosons provides a concrete example of this. The phenomenology of KK-excitations has been studied in great detail in recent times which covers its implications at colliders [3, 4, 5, 6, 7, 8, 9, 10, 11, 12, 13, 14, 15, 16], in electroweak/flavour sector via various low-energy observables [17, 18, 19, 20, 21, 22, 23, 24, 25, 26, 27, 28] and for dark matter/cosmology [5, 13, 29, 30, 31, 32, 33, 34, 35, 36, 37, 38, 39, 40]

In MUED there are only two extra free parameters when compared to the SM. These are the radius of compactification $R$ (or, alternately $R^{-1}$, the scale of compactification) and the cutoff scale $\Lambda$ of the theory. The MUED parameter space also includes the

SM Higgs mass $m_{H_{S M}}$. It is thus noteworthy that MUED is much more economic than even the most constrained version of SUSY scenarios, like the minimal supergravity (mSUGRA) framework.

It is to be noted that the phenomenology of MUED turns out to be remarkably similar and thus can masquerade as SUSY [6]. This is because of two basic reasons. First, both frameworks predict new excitations bearing the same gauge and global quantum 
numbers corresponding to the SM particles. Second, in each of these scenarios there is a conserved discrete symmetry: the so-called $R$-parity in the SUSY case while its counterpart in the UED scenario is the KK-parity, $K_{P}=(-1)^{n}$, where $n$ stands for the $n$-th level excitation. Conservation of KK-parity is very central to the phenomenology of MUED and is similar in its ramifications to $R$-parity of SUSY scenarios. Vigorous efforts are on [8, 11, 14, 41, 42, 43, 44, 45, 47, 46, 48, 49, 50, 51, 52, 53, 54, 55, 56, 57] in chalking out strategies and their respective efficiencies to distinguish between these two contending scenarios under different situations. These efforts broadly exploits two salient differences between the two scenarios. While in the case of MUED the KKexcitations all have the same spin as their SM counterparts, for SUSY they differ by half. Also, MUED spectrum is comprised of a tower of discrete KK-excitations of the SM particles unlike the SUSY spectrum.

As does $R$-parity for SUSY, KK-parity forbids tree-level contribution to weak-scale processes. In some earlier works it was reported that the electroweak precision data require a lower bound of $R^{-1} \gtrsim 250-300 \mathrm{GeV}$ [1, 58. This finding was later shown to be consistent with observations in the $B$-physics sector [17] and with the experimental results on anomalous magnetic moments [18, 19]. However, in later years, with more data and newer analyses it has been observed that the lower bound on $R^{-1}$ can surpass the earlier value and can be as high as $700 \mathrm{GeV}$ [27, 39, 59] at 99\% confidence level. Also, similar to $R$-parity in SUSY scenarios, KK-parity ensures that the lightest $n=1$ KK-paricle (the LKP, with odd KK-parity) is an absolutely stable (like the lightest SUSY particle, the LSP) weakly interacting massive particle (WIMP) and is a good dark matter candidate that could provide the right amount of cosmological relic-density [31]5. Given the allowed mass-scale as low as few hundred $\mathrm{GeV}$ and with a viable dark matter candidate in its spectrum, the scenario thus offers exciting signatures at future generation colliders, particularly at the LHC (see references [3]-[15]).

Interestingly enough, in contrast to a huge amount of recent and on-going activities in so many different aspects of the MUED-phenomenology, the collider aspects of the Higgs sector of the MUED has not been discussed in any detail until very recently. The MUED Higgs sector was discussed in [37, 38] in the context of estimating the relic abundance of KK-dark matter. In ref. [60] the authors explore some characteristic regions of the MUED parameter space and their general bearings on the MUED Higgs phenomenology including those at the colliders (with rather characteristic and novel signatures). To the best of our knowledge, dedicated and somewhat detailed collider

\footnotetext{
${ }^{5}$ Usually, the level $1 \mathrm{KK}$-excitation of the $U(1)$ gauge field $B$ of the SM, $B_{1}$, turns out to be the LKP in the MUED scenario. The Weinberg angle at higher KK-levels being very small [5] $B_{1}$ is sometimes identified as the lightest KK-photon in the literature. In Ref. [60], however, possibilities of other level-1 excitations like the charged Higgs boson (see section 2) and the excited tau lepton becoming the LKP were discussed in some detail.
} 
studies of the MUED Higgs bosons were only taken up in refs. [61, 62] 6 .

In the present work, we study the cascade decays of the level-1 MUED excitation as one of the major sources for the KK level-1 Higgs bosons of the MUED with $B_{1}$ as the LKP. We also look at the dominant decay modes of the KK Higgs excitations thus identifying the final states in which they can be searched for at the LHC. Also, for the first time ever, the full mixing in the third generation level-1 fermion sector is taken into account and applied to collider studies. The mixings are phenomenologically less significant in KK level-1 tau lepton and bottom quark sectors when compared to the corresponding top-quark sector.

The paper is organized as follows. In section 2 we briefly outline the MUED framework a la ACD [1] with particular emphasis on its Higgs sector [21. The spectrum of Higgs bosons and its nature and dependencies which are crucial for their studies at the LHC are discussed. In this work, we restrict ourselves to the physics of the level-1 charged Higgs boson of the MUED. In section 3 we describe the possible decays of the KK-Higgs bosons under different circumstances and their implications at the colliders. Section 4 deals with different production mechanisms for the KK-Higgs bosons and identify the significant ones at the LHC. We also present the results of our numerical analysis in terms of the signal-strength and tentative reach in $R^{-1}$. Section 5 summarizes with an outlook that touches upon issues that emerge in the process.

\section{The Higgs sector of the minimal UED}

The structure of the fermion and the gauge boson sectors of the MUED have been discussed in the literature in rather details [1, 67]. The corresponding mass-spectra have also been discussed extensively. It is also established that radiative corrections [5] to the masses of these KK-excitations play crucial roles in the phenomenology of the MUED by lifting the degeneracy in their masses which otherwise, at tree level, go as $n R^{-1}$ where $n$ stands for the concerned level in the tower of KK-excitations.

However, as pointed out in the Introduction, the structure and properties of the Higgs sector of the MUED and the consequent phenomenological implications it bears have not received enough attention (except in the references mentioned earlier). So much so that none of the popular packages had yet included the Higgs sector in their MUED implementations [67, 68, 69] In the following subsections we outline the basic

\footnotetext{
${ }^{6}$ Another set of literature 63, 64, 65, 66, discuss the virtual effects of the MUED states on Higgs physics at future colliders including the LHC and the extent of their detectability. These studies exploit Higgs boson production via gluon fusion at the LHC and its decay in the two-photon final state. It was observed in ref. 65] that the uncertainties involved, when combined, could potentially dilute the deviations from the SM to an insignificant level unless one has a better control on this uncertainties. In ref. 66] it is observed that these virtual effects of the MUED may cease to be perceptible at the same time when the MUED excitations become heavy enough to evade direct discovery at the LHC.
} 
construct of the MUED Higgs sector which involves the gauge boson sector in a very characteristic way. Also, the Higgs-phenomenology in the MUED involves the KKfermions and the gauge bosons in a crucial manner. We would highlight them in appropriate contexts. We would then point out some features and interplays that make the Higgs sector of the MUED rather special.

\subsection{The basic construct}

In the MUED with $(4+1)$ dimensions we are confronted with two well-known but fundamental problems. First, we cannot obtain chiral fermions which we find in nature. Second, the 5th components of the (4+1)-dimensional gauge fields behave as some weird CP-odd scalars in $(3+1)$ dimensions which we do not see in nature. In the framework of MUED, in order to accommodate the chiral fermions in the theory and to project out the unwanted scalar degrees of freedom, one seeks an orbifold compactification of the kind $S^{1} / Z_{2}$ for the extra space dimension (say, denoted by $y$ ), i.e., compactifying it on a circle $S^{1}$ (a sphere of genus 1 ) about an orbifold identified with two diametrically opposite fixed points $y=0, \pi R$ (a $Z_{2}$ symmetry dubbed the KK-parity) along the direction $y$ [1], $R$ being the radius of compactification. A framework like MUED with a small $\left(R^{-1} \sim \mathcal{O}(\mathrm{TeV})\right)$ but flat extra dimension thus would likely to have interesting implications for the LHC and their studies have attracted significant attention in recent times as indicated in section 1.

The boundary conditions imposed at the two orbifold fixed points determine the KK expansion for different fields. A scalar field has to be either odd or even under the transformation $\mathcal{P}: y \rightarrow-y$ at $y=0, \pi R$. Thus, $\partial_{5} \phi^{+}=0$ for the even fields (the Neumann boundary condition) and $\phi^{-}=0$ for the odd fields (the Dirichlet boundary condition) at the fixed points. The associated Fourier expansions of $(4+1)$-dimensional scalar fields in terms of the $(3+1)$-dimensional KK-modes look like

$$
\begin{array}{ll}
\phi^{+}\left(x^{\mu}, y\right)= & \frac{1}{\sqrt{2 \pi R}} \phi_{(0)}^{+}\left(x^{\mu}\right)+\frac{1}{\sqrt{\pi R}} \sum_{n=1}^{\infty} \phi_{(n)}^{+}\left(x^{\mu}\right) \cos \frac{n y}{R} \\
\phi^{-}\left(x^{\mu}, y\right) & = \\
& \frac{1}{\sqrt{\pi R}} \sum_{n=1}^{\infty} \phi_{(n)}^{+}\left(x^{\mu}\right) \sin \frac{n y}{R}
\end{array}
$$

where $\mu=0,1,2,3$ stands for the four ordinary non-compact space-time coordinates and $\phi_{(n)}^{ \pm}\left(x^{\mu}\right)$ are the $(3+1)$ dimensional KK-modes with $n=0$ (level 0 ) standing for the SM excitation. Clearly, by construct and as required, a $Z_{2}$-odd state at level ' 0 ' is projected out by the choice of boundary conditions. The odd degrees of freedom start appearing only from level ' 1 ' and hence, they do not have any corresponding SM field.

On the other hand, a vector field $A^{M}$ possesses 5 components in $(4+1)$ dimensions, i.e., $\mathrm{M}=0,1,2,3$ and 5 , conventionally. The orbifold compactification of the form $\partial_{5} A^{\mu}=0$ along with $A^{5}=0$ ensures that the first 4 components are even under $\mathcal{P}_{5}$ while 
the 5-th component transforms as an odd degree of freedom. The Fourier expansions of the $(4+1)$ dimensional vector field in terms of corresponding $(3+1)$ dimensional KK-modes are

$$
\begin{array}{ll}
A^{\mu}\left(x^{\mu}, y\right)= & \frac{1}{\sqrt{2 \pi R}} A_{(0)}^{\mu}\left(x^{\mu}\right)+\frac{1}{\sqrt{\pi R}} \sum_{n=1}^{\infty} A_{(n)}^{\mu}\left(x^{\mu}\right) \cos \frac{n y}{R} \\
A_{5}\left(x^{\mu}, y\right)= & \frac{1}{\sqrt{\pi R}} \sum_{n=1}^{\infty} A_{(n)}^{5}\left(x^{\mu}\right) \sin \frac{n y}{R}
\end{array}
$$

As in the case for the scalars above, here also, the $Z_{2}$-odd gauge degrees of freedom which behave as scalars in $(3+1)$ dimensional description appear only from the first KK level and thus have no corresponding SM excitation. These extra $Z_{2}$-odd scalar degrees of freedom in equations 1 and 2 may combine to give new scalar degrees of freedom at level 1 onwards.

Similarly, with appropriate boundary conditions imposed at the orbifold fixedpoints the expansion of the the $(4+1)$ dimensional 4-component Dirac fermions are

$$
\begin{aligned}
& \psi^{+}\left(x^{\mu}, y\right)=\frac{1}{\sqrt{2 \pi R}} \psi_{R(0)}\left(x^{\mu}\right)+\frac{1}{\pi R} \sum_{n=1}^{\infty}\left(\psi_{R(n)}\left(x^{\mu}\right) \cos \frac{n y}{R}+\psi_{L(n)}\left(x^{\mu}\right) \sin \frac{n y}{R}\right) \\
& \psi^{-}\left(x^{\mu}, y\right)=\frac{1}{\sqrt{2 \pi R}} \psi_{L(0)}\left(x^{\mu}\right)+\frac{1}{\pi R} \sum_{n=1}^{\infty}\left(\psi_{L(n)}\left(x^{\mu}\right) \cos \frac{n y}{R}+\psi_{R(n)}\left(x^{\mu}\right) \sin \frac{n y}{R}(3)\right)
\end{aligned}
$$

As should be the case, the zero-modes, i.e., the SM fermions are either left or right handed. Thus, the chiral fermions of the SM are projected in by the orbifolding while all the excited KK fermions are made up of chiral pairs, i.e., these are vector-like fermions. In other words, while the SM fermions are constructed out of chiral components which have different $S U(2)_{L} \times U(1)_{Y}$ quantum numbers, in the MUED the components have the corresponding quantum numbers the same. This difference is phenomenologically crucial and shows up in the couplings of these vector like states with the gauge bosons and the Higgs bosons of the MUED.

We now collect the components that build up the MUED Higgs sector. Since in UED all SM excitations including the Higgs degrees of freedom can propagate in the 5 -dimensional bulk, on compactification, these also get a tower of scalars. The KK excitations of the components of the SM Higgs doublet can be expressed using the usual compact notation:

$$
H_{(n)}=\left(\begin{array}{c}
i \chi_{(n)}^{+} \\
\frac{1}{\sqrt{2}}\left(h_{(n)}+i \chi_{(n)}\right)
\end{array}\right)
$$

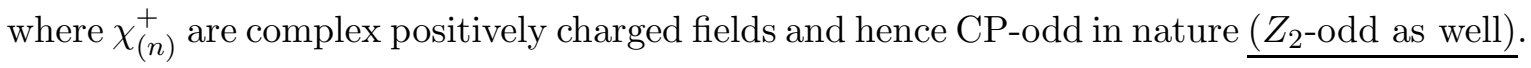
On the other hand, starting KK level 1, we already have the 5th components of the electroweak gauge bosons which behave as $\mathrm{CP}\left(Z_{2}\right)$-odd scalars on compactification to $(3+1)$ dimensions. These excitations are $B_{(n)}^{5}, W_{3(n)}^{5}$ and $W_{(n)}^{1,2(5)}$. Note that these 
scalar degrees of freedom are not exact extra-dimensional analogues of the SM NambuGoldstone bosons since they all receive the KK-mass of $\frac{n}{R}$ because of the derivative operator $\partial_{5}$ operating on them. When the electroweak symmetry is broken $B_{(n)}^{5}$ and $W_{3(n)}^{5}$ mix to give $\gamma_{(n)}^{5}$ and $Z_{(n)}^{5}$ while $W_{(n)}^{1(5)}$ and $W_{(n)}^{2(5)}$ combine to result in $W_{(n)}^{ \pm(5)}$ analogous to what happens in the SM.

Now, $Z_{(n)}^{5}$ mixes with $\chi_{(n)}$ while $W_{(n)}^{ \pm 5}$ mixes with $\chi_{(n)}^{ \pm}$to form not only the effective Nambu-Goldstone bosons $G_{(n)}^{0}$ and $G_{(n)}^{ \pm}$but also the three physical Higgs bosons, the CP-odd neutral $A_{(n)}^{0}$ and the charged Higgs bosons $H_{(n)}^{ \pm}$. The Goldstone states $G_{(n)}^{0}$ and $G_{(n)}^{ \pm}$are absorbed by the SM gauge bosons to become massive keeping the gauge invariance intact. The mutually orthogonal Goldstone and physical Higgs boson states are given by

$$
\begin{array}{clrl}
G_{(n)}^{0}=\frac{1}{M_{Z_{(n)}}}\left[M_{Z} \chi_{(n)}-\frac{n}{R} Z_{(n)}^{5}\right] & A_{(n)}^{0}=\frac{1}{M_{Z_{(n)}}}\left[\frac{n}{R} \chi_{(n)}+M_{Z} Z_{(n)}^{5}\right] \\
G_{(n)}^{ \pm}=\frac{1}{M_{W_{(n)}}}\left[M_{W} \chi_{(n)}^{ \pm}-\frac{n}{R} W_{(n)}^{ \pm 5}\right] & A_{(n)}^{ \pm}=\frac{1}{M_{W_{(n)}}}\left[\frac{n}{R} \chi_{(n)}^{ \pm}+M_{W} W_{(n)}^{ \pm 5}\right]
\end{array}
$$

where $M_{Z_{(n)}}$ and $M_{W_{(n)}}$ are given by

$$
M_{Z_{(n)}}^{2}=\frac{n^{2}}{R^{2}}+M_{Z}^{2} \quad M_{W_{(n)}}^{2}=\frac{n^{2}}{R^{2}}+M_{W}^{2}
$$

Also, note that the KK excitations of the SM Higgs boson is physical CP-even states which do not mix with the CP-odd $\gamma_{(n)}^{5}$ states. The latter remain to be additional unphysical scalar modes which act as Goldstone bosons absorbing which $\gamma_{(n)}^{\mu}$ become massive for $n \geq 1$. As implied by equation 2 , $\gamma^{5}$ would not have ' 0 ' modes which thus keeps the usual photon (level ' 0 ') massless.

It is evident from equation 6 that with increasing ' $n$ ', i.e., at higher KK-levels, the Goldstone modes are dominated by $Z_{(n)}^{5}$ and $W_{(n)}^{ \pm 5}$ while the physical Higgs modes are mostly $\chi_{(n)}$ and $\chi_{(n)}^{ \pm}$. Taking a cue from equation 1 it is extremely crucial to note that the Higgs states $A_{(n)}$ and $H_{(n)}^{ \pm}$do not have a ' 0 ' mode. They first appear at the KK level 1. To summarise, there are 4 new Higgs states at KK level 1 which are $A_{(n)}, H_{(n)}^{ \pm}$ and $H_{(n)}$ of which only the last one has a level ' 0 ' mate which is the usual SM Higgs boson. Thus, if we restrict ourselves to KK level-1 for studying the Higgs bosons, we end up with 5 Higgs bosons in total of which only one (the level '0' (SM) Higgs boson) has even KK-parity while the other four from level 1 are of odd KK-parity.

The interesting thing to note here is that the number of Higgs bosons (up to KK level 1) in the MUED framework is exactly the same as that in the Minimal Supersymmetric Standard Model (MSSM). Thus, much talked about faking of the MSSM by a MUED like scenario encompassing the fermion and the gauge boson sectors, comes to a full circle with the similarity in the scalar sectors that unfolds. The origins of the scalar states in the two scenarios, however, are in clear contrast. The five Higgs bosons of the MSSM are the well-known outcome of a generic scenario with two Higgs doublets. 
They all are $R$-parity even. In contrast, in the MUED, the only (SM) Higgs doublet suffices. There, the multiplicity of the Higgs states is traced back to the subtle presence of KK towers for the additional (scalar) degrees of freedom pertaining to the gauge sector that result from orbifolding. Also, only one of these Higgs bosons (the level- ' 0 ' or the SM one) carries even KK-parity while the other four are odd under the same. This feature is particularly crucial for the phenomenology of the Higgs bosons in the MUED where KK-parity is conserved. It is not at all unexpected if we remember what role $R$-parity conservation plays in shaping the SUSY phenomenology.

\subsection{The mass spectrum and the mass-splittings}

In this subsection we discuss the Higgs mass spectrum of the MUED vis-a-vis the spectrum of other MUED excitations and reflect on their phenomenological implications.

The Higgs spectrum of the MUED at KK level $n$ is determined by the following relations:

$$
\begin{aligned}
& m_{H_{n}^{0}}=m_{n}^{2}+m_{H}^{2}+\hat{\delta} m_{H_{n}}^{2} \\
& m_{A_{n}^{0}}=m_{n}^{2}+m_{Z}^{2}+\hat{\delta} m_{H_{n}}^{2} \\
& m_{H_{n}^{ \pm}}=m_{n}^{2}+m_{W}^{2}+\hat{\delta} m_{H_{n}}^{2}
\end{aligned}
$$

where, $m_{H}$ is the mass of the SM Higgs boson and $\hat{\delta} m_{H_{n}}^{2}$ represents the total oneloop correction (including both bulk and boundary contributions, the former being identically equal to zero in the case of Higgs bosons) which is universal to all the Higgs states at level 1 and is given by [5]

$$
\hat{\delta} m_{H_{n}}^{2}=m_{n}^{2}\left(\frac{3}{2} g_{2}^{2}+\frac{3}{4} g^{\prime 2}-\lambda_{H}\right) \frac{1}{16 \pi^{2}} \ln \frac{\Lambda^{2}}{\mu^{2}}+\bar{m}_{H}^{2}
$$

where $m_{n}$ refers to the universal KK mass arising out of compactification at level $n$ and given by $\frac{n}{R}, g_{2}$ and $g^{\prime}$ stand for the $S U(2)$ and the $U(1)$ gauge couplings respectively, $\lambda_{H}$ represents the standard Higgs quartic coupling and is given by $\lambda_{H}=m_{H}^{2} / v^{2}, \Lambda$ gives the cut-off scale $(\mathcal{O}(\mathrm{TeV}))$ up to which the UED description is valid, $\mu$ stands for the renormalization scale and $\bar{m}_{H}^{2}$ is the universal boundary mass term for the Higgs mode which is a free parameter set to zero in the MUED but we shall also look into the implications of non-zero values for the same. Thus, the Higgs sector of the MUED is completely specified by the following free parameters: $\Lambda, R^{-1}, m_{H}, \mu$ and $\bar{m}_{H}^{2}$.

From equations 7 and 8 it is apparent that the hierarchy of the 4 Higgs boson masses at the first KK level is as follows:

$$
m_{H_{1}^{ \pm}}<m_{A_{1}^{0}}<m_{H_{1}^{0}}
$$

It is also rather clear that the terms responsible for the mass-splittings among the KKHiggs masses are those involving the masses of the SM gauge bosons and the SM Higgs 
boson. $M_{W}$ and $M_{Z}$ being the experimentally measured quantities, the individual masses of the level-1 charged and the CP-odd neutral Higgs bosons (as well as their mutual splittings) are pretty much determined. $m_{H_{1}^{0}}$, however, remains a function of the unknown SM Higgs boson mass but guaranteed to be the heaviest of them all with $m_{H}$ (i.e., the KK level-0 Higgs boson which is the SM Higgs boson) satisfying the LEP bound (i.e., $m_{H} \gtrsim 114.4 \mathrm{GeV}$ ) [77]. It is interesting to note that with increasing $m_{H}$, $\lambda_{H}$ increases (in equation (8) thus decreasing $\hat{\delta} m_{H_{n}^{2}}$ which results in decreasing $m_{H_{1}^{0}}$. Also, with increasing $R^{-1}$, the masses of all the KK Higgs bosons get dominated by the KK-mass term $(n / R)$ and the mass-splittings among the excited Higgs bosons become increasingly small. However, phenomenologically, the more relevant parameters are the splittings among the KK Higgs bosons and the low-lying KK excitations like the singlet KK leptons and the lightest KK particle (LKP) in which the former could decay. In section 3, we illustrate a representative situation and its phenomenological implications.

\section{Decay of the level-1 charged Higgs boson}

In this section, we discuss the possible decay modes of the level-1 charged Higgs boson and the expected features in the kinematics of its decay products. It is clear from the last section that the KK Higgs bosons are some of the lowest lying states in the MUED spectrum. With $\bar{m}_{H}^{2}$ set to zero, only $B_{1}$ (the LKP), the KK leptons and the KK neutrinos can become lighter than the level-1 charged KK Higgs boson $\left(H_{1}^{ \pm}\right)$ depending upon the value of $R^{-1}$. However, for larger $R^{-1}$ (as would be demonstrated later in this section) only singlet type KK-leptons could become lighter than $H_{1}^{ \pm}$and thus, are the states to which the latter can favourably decay into.

There are thus a few characteristic modes in which $H_{1}^{ \pm}$can decay depending upon the spectrum of the above mentioned excitations. In the MUED, with varying input parameters (in particular, $R^{-1}$ ), there can be the following two phenomenologically relevant mass-orderings involving the above-mentioned low-lying MUED excitations which are somewhat degenerate in nature:

(i) $m_{B_{1}}<m_{\ell_{1}^{S}}<m_{H_{1}^{ \pm}}<m_{\ell_{1}^{D}}$ : In this case, $H_{1}^{ \pm}$would undergo the two-body decay

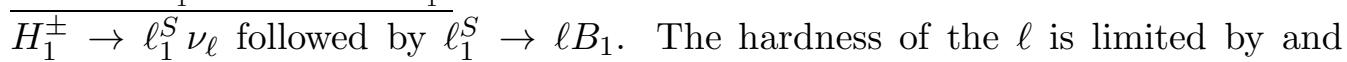
increases with the mass-splitting $\left(\Delta m_{\left(\ell_{1}^{S} B_{1}\right)}\right)$ between $\ell_{1}^{S}$ and $B_{1}$. From Fig. 1 it is clear that the mass-split in reference and hence the hardness of the lepton would increase with increasing $R^{-1}$. Thus, for $500 \mathrm{GeV}<R^{-1}<2000 \mathrm{GeV}$, one can expect $p_{T}^{\ell}$ to range between $15 \mathrm{GeV}<p_{T}^{\ell}<25 \mathrm{GeV}$. Detection of such a soft lepton would be a challenge at the LHC. Nevertheless, uncovering scenarios with such generic degeneracies in the spectrum would necessarily require such a 
resolution 7 .

(ii) $m_{B_{1}}<m_{H_{1}^{ \pm}}<m_{\ell_{1}^{S}}<m_{\ell_{1}^{D}}$ : Here, $H_{1}^{ \pm}$is the next-to-lightest KK particle (NLKP) 8 $H_{1}^{ \pm}$could only undergo 3-body decays of the form $H_{1}^{ \pm} \stackrel{q_{1}^{*}, \ell_{1}^{*}}{\longrightarrow} f \bar{f}^{\prime} B_{1}$ via off-shell quarks and leptons. It has earlier been observed in ref. 60] that the decay lengths can be as big as $20 \mathrm{~cm}$ for $\Delta m=1 \mathrm{GeV}$ and for $\Delta m \lesssim 0.4 \mathrm{GeV} H_{1}^{ \pm}$becomes essentially stable at colliders. These situations could result in displaced vertices and non-zero impact parameters, signals of slow and metastable charged with highly ionizing tracks etc. However, not the individual but the combined hardness of the SM fermions (quarks or lepton and neutrino) in the final state is governed by the mass-splitting $\left(\Delta m_{\left(H_{1}^{ \pm} B_{1}\right)}\right)$ between $m_{H_{1}^{ \pm}}$and $B_{1}$. This mutual sharing would render the final-state fermions even softer, especially when the mass splitting is already miniscule, as is the case here. It will be a challenge at the LHC (or, for that matter, at any future collider) to detect such soft fermions and hence the signatures that depend critically on this.

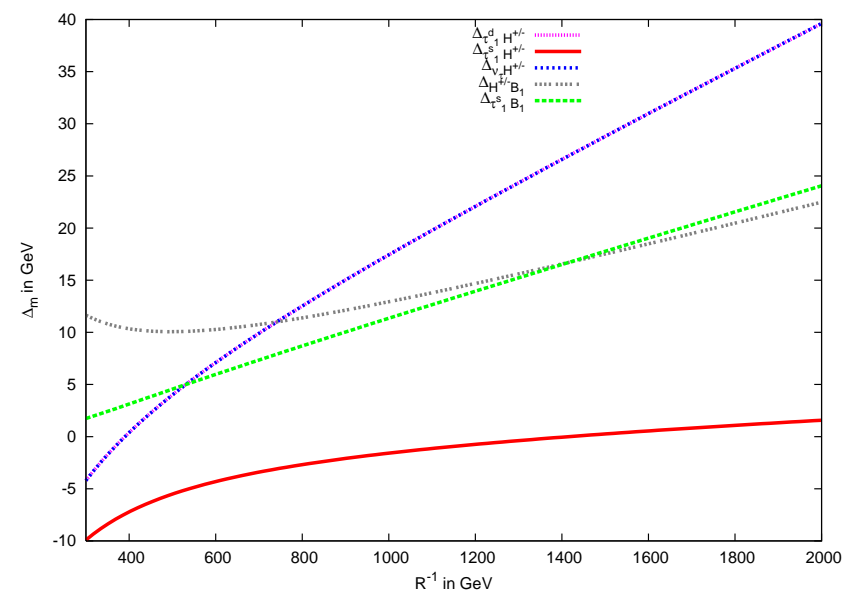

Figure 1: Variation of the mass-splittings between different low-lying level-1 KK-excitations of the MUED as functions of $R^{-1}$ and for $\Lambda R=20$ and $m_{H_{S M}}=120 \mathrm{GeV}$. Convention followed for the legend is that the mass of the second particle is subtracted from that of the first.

Some crucial observations worth special mentions:

- First, although the fermion detection efficiencies would increase with increasing $R^{-1}$ (at least for extreme to moderately soft fermions), the optimal reach in the latter via search for $H_{1}^{ \pm}$would be determined by how sharply the production cross section of $H_{1}^{ \pm}$drops with $R^{-1}$.

\footnotetext{
${ }^{7}$ A similar situation occurs in a supersymmetric scenario in the so-called stau coannihilation region where the stau and the LSP (the lightest neutralino) are rather degenerate [78, 79].

${ }^{8}$ Phase 2 of the phase diagram in ref. $[60]$.
} 
- Second, in the two cases discussed above, the degeneracy of the mother and the daughter (KK) excitations are such that in the first case the MUED excitations would decay promptly while the latter one is likely to have exotic signals from long-lived, metastable excitations. The region of parameter space where such phenomenon may occur depends crucially on the mass of the SM Higgs boson 60]. In our analysis, however, we have kept $m_{H_{S M}}$ fixed at $120 \mathrm{GeV}$ for which the latter phase takes over at around $R^{-1}=1400 \mathrm{GeV}$ (see the bottom-most curve of Fig. 1.

- Lastly, the LKP, though by virtue of being rather heavy $\left(m_{B_{1}} \sim R^{-1}\right)$ could carry a lot of $p_{T}$, the vector-sum of the same for two of them 9 in the final state may suffer significant cancellation because of the afore-mentioned mass-degeneracy between the decaying particle and the the LKP10. Thus, the missing $p_{T}$ in any event turns out to be characteristically soft in contrary to the generic expectation. These are the kinematic regimes where the backgrounds are generally very high thus making the hunt for exotics like $H_{1}^{ \pm}$further challenging. A detailed simulation with major detector effects incorporated would be required for a reasonable understanding of the phenomenon.

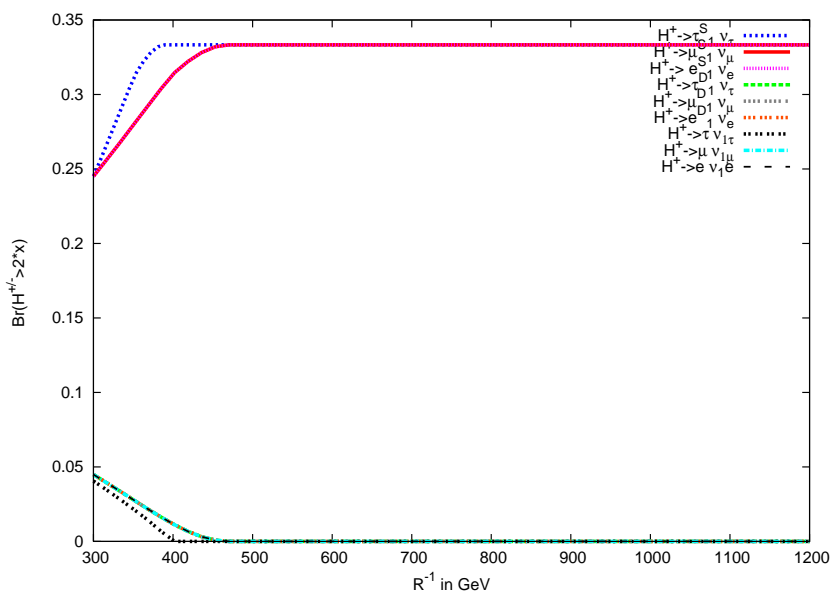

Figure 2: Variation of the decay branching fractions of the level-1 charged Higgs boson $\left(H_{1}^{ \pm}\right)$to leptons and neutrinos as a function of $R^{-1}$ (in $\mathrm{GeV}$ ). The blue (top) curve stands for the combined branching fraction of $H_{1}^{ \pm}$to singlet level- $1 \mathrm{KK}$ leptons while the red and black (bottom) correspond to the combined fractions to doublet level-1 KK leptons and level-1 KK-neutrinos respectively.

Consequently, searches for the KK Higgs bosons are supposed to be heavily plagued by the background thus making their observation extremely difficult at any future

\footnotetext{
${ }^{9}$ Note that, similar to SUSY scenarios with conserved $R$-parity, KK-parity in MUED ensures that level-1 MUED states would be pair-produced at the LHC and they would ultimately cascade to a pair of LKPs $\left(B_{1}\right.$, in our case).

${ }^{10}$ Similar observations were made in Refs. [71, 72].
} 
collider including the LHC. In Fig. 2 we present the branching fractions of the charged level-1 KK-Higgs boson to level-1 KK leptons and KK neutrinos. The behaviour of the curves can be explained with the help of the expressions for the respective couplings as given in ref. 21]. However, the interplay of different parameters that are instrumental is quite complex. One can, however, try to follow the proceedings in a somewhat intuitive way as follows.

Note that the couplings of a pure KK Higgs boson to the KK leptons and/or KK neutrinos necessarily involve a chirality-flip. Since, a neutrino (or its KK excitation) is a helicity eigenstate (left-chiral), its interaction vertex with a 'pure' charged Higgs boson could only involve a right chiral charged KK lepton (or its SM counterpart) whose strength is proportional to the lepton Yukawa coupling and is, in turn, proportional to its mass. This issue is clear if we note that the decay branching fraction to $\tau_{1}^{S}$ is a little more enhanced that that for the other two lepton flavours for low $R^{-1}$.

The overwhelmingly large branching fraction to singlet KK-leptons is due to the availability of phase space while the same gets closed for $H_{1}^{ \pm} \rightarrow \ell_{1}^{D} \nu$ for $R^{-1} \gtrsim 400$ GeV since $m_{\ell_{1}^{D}}>m_{H_{1}^{ \pm}}$for such $R^{-1}$ (see Fig. 1). Once they lead, the individual branching fractions to $e_{1}^{S}, \mu_{1}^{S}$ and $\tau_{1}^{S}$ are mainly governed by the term proportional to the respective fermion masses for low $R^{-1}$. It should be remembered that the absolute width for decays of $H_{1}^{ \pm}$in these modes are still small $\left(\lesssim \mathcal{O}\left(10^{-5} \mathrm{GeV}\right)\right)$ though ensures promptness of its decay. With growing $R^{-1}$ the terms in the coupling conspire to ensure a universality among the three lepton flavours in the decay of $H_{1}^{ \pm}$. This feature goes a long way in shaping the phenomenology at the LHC. In regions of MUED parameter space where $\ell_{1}^{S}$-s are the NLKP $H_{1}^{ \pm}$decays almost indiscriminately to all three flavours. With $p_{T}^{\ell}$ being governed by the mass-split $\Delta m_{\ell_{1}^{S} B_{1}}$ (from the subsequent decays of $\ell_{1}^{S} \rightarrow \ell B_{1}$ ) it is expected (from Fig. 1) that the former can attain a value of around $15 \mathrm{GeV}$ before a new phase emerges at around $1.4 \mathrm{TeV}$. The important point to note here is that one may try to tag the soft electrons and muons which is comparatively easier instead of looking for such a soft tau.

However, as the charged Higgs boson (as well as all the excited Higgs bosons) has an admixture of 5th (scalar) component of the 5-dimensional gauge bosons, this may lead to some chirality-conserving (gauge) couplings albeit suppressed by the mass of the appropriate KK gauge boson. This feature is clear from the fact that there are some small but non-zero branching fractions to doublet KK-leptons and to KK-neutrinos at relatively small $R^{-1}$ which quickly (at around $R^{-1}=400 \mathrm{GeV}$ ) drops to insignificant levels as $R^{-1}$ grows.

In this context, it should be mentioned that with increasing mass of the SM Higgs boson, $H_{1}^{ \pm}$may become the LKP for larger values of $R^{-1}$. Such a phase raises the standard debates pertaining to the implications of a stable charged particle as the dark matter candidate and a clarification to this is given in 60]. We, however, do not get into the analysis of this phase at this stage. 


\section{Production of level-1 KK Higgs bosons}

It is rather obvious from the previous section that finding the KK Higgs bosons at colliders would be a difficult task due to the kinematic features (softness) their decay products bear. Thus, any serious attempt to resurrect the possibility of finding the KK Higgs boson should, in the first place, ensure that the production cross section of them are reasonably high so as to make up for a low overall efficiency that would be plaguing their detection. Hence, in this section, we take up a brief study on the production rates of the KK Higgs bosons (particularly, for the charged KK Higgs boson).

Level-1 KK Higgs bosons have odd KK-parity and hence cannot be produced as a single resonance unlike the Higgs bosons of the SM or those of the SUSY scenarios which have even $R$-parity. KK Higgs bosons can only be produced in association with another level-1 KK excitation which has a similar mass as the KK Higgs boson $\left(\sim R^{-1}\right.$. This brings in a significant demand on the available phase space when compared to the cases of Higgs boson production in SM and the SUSY scenarios where not only their resonant productions but also their associated productions with lighter particles like the SM gauge bosons and fermions are promising.

The production processes of the level-1 KK Higgs bosons can be broadly divided into two categories: (i) direct production processes and (ii) production under UED cascade decays. The direct production processes include pair production of the level-1 Higgs bosons and their production in association with level-1 gauge bosons like $Z_{1}$ and $W_{1}^{ \pm}$. It is already well-known that the rates for these processes are not at all significant at the LHC. This is not unexpected since these involve production of two massive $\sim R^{-1}$ particles mainly driven by weak interaction. We leave the detail exploration of such processes for a future work. Pair production of $t_{1} \bar{t}_{1}$ followed by their decays to charged level-1 KK-Higgs bosons was discussed in Ref. 62]. In the present work, we take a special note of the couplings involved in the latter processes and concentrate on the production of the level-1 charged KK-Higgs bosons under cascades (involving UED excitations) at the LHC. This is in line with and motivated by similar studies undertaken for the MSSM Higgs bosons in recent times and which turned out to be very promising 11 [73, 74].

\footnotetext{
${ }^{11}$ This is a possibility as it is well-known that corresponding to each cascade diagram involving SUSY particles in the MSSM there is a corresponding "twin diagram" 11 involving their KK-counterparts in MUED. While for the MSSM Higgs bosons these may not serve as the discovery channels but would play complementary and/or supplementary roles in deciphering the MSSM Higgs system, in case of the level-1 KK Higgs bosons these turn out to be the only modes that are phenomenologically interesting.
} 


\subsection{Production of $H_{1}^{ \pm}$under UED cascades}

The production of $H_{1}^{ \pm}$under UED cascades takes advantage of the large production cross section of the strongly interacting UED excitations (level-1 KK gluon and quarks) at the LHC. The yield of $H_{1}^{ \pm}$under cascade decays of these excitations depends crucially on how favourably they decay to $H_{1}^{ \pm}$. To this effect, pair-production of level-1 KK gluon $\left(p p \rightarrow g_{1} g_{1}\right)$ and the associated productions of level-1 gluon and level-1 quarks $\left(p p \rightarrow g_{1} q_{1}\right)$ play the dominant roles. For these, it is the effective decay branching fraction of $g_{1}$ to $H_{1}^{ \pm}$that holds the key. Decays of $q_{1}$ to $H_{1}^{ \pm}$are suppressed for the first two generations of quarks and are only significant for the ones from the third generation, i.e., for $b_{1}$ and $t_{1} 12$ Thus, pair productions of the third generation level-1 KK quarks have somewhat significant contributions to $H_{1}^{ \pm}$through their decays. In fact, to obtain $H_{1}^{ \pm}$from the decay of $g_{1}$, the cascades almost necessarily have to go via an intermediate state involving a third generation level-1 quark, i.e., $g_{1} \rightarrow b(t) \bar{b}_{1}\left(\bar{t}_{1}\right) \rightarrow$ $H_{1}^{ \pm}+X$. Hence, an important part of the present work is the study of the decays of level-1 gluon to $b_{1}$ and $t_{1}$ and their subsequent decays to $H_{1}^{ \pm}$. We reiterate that $(S U(2)$ and $U(1))$ mixing in the third generation quark and lepton sector has been fully incorporated in our present analysis. It is observed that the lighter mass eigenstates $t_{1}$ and $b_{1}$ have predominantly the $S U(2)$ component while the heavier $t_{2}$ and $b_{2}$ states are mostly of $U(1)$ type. These relative strengths of different couplings play crucial roles in subsequent analysis.

The couplings that are instrumental in determining the strengths of different branching modes of $t_{1}^{(1,2)}$ and $b_{1}^{(1,2)}$ to $H_{1}^{ \pm}$are $H_{1}^{+} \bar{t}_{1} b, H_{1}^{+} \bar{t}_{2} b, H_{1}^{-} \bar{b}_{1} t, H_{1}^{-} \bar{b}_{2} t$ (and their hermitian conjugates). We checked and used these couplings as given in ref. [21]. A minute inspection of these couplings reveals that the $U(1)$ type (heavier) level-1 top quark has a coupling with $H_{1}^{ \pm}$which is similar to the coupling involving the $S U(2)$ type (lighter) level-1 KK bottom quark and $H_{1}^{ \pm}$. These couplings are enhanced compared to the other set of similar couplings relevant in the present analysis, i.e., the couplings of $H_{1}^{ \pm}$with lighter level-1 KK top quark and that with the heavier level-1 KK bottom quark. As we would see, these relative strengths of different couplings play crucial roles in subsequent analysis.

For numerical purposes we use CalcHEP 68 and modified the available model files [67] for MUED to incorporate the Higgs sector up to KK level-1 by including the couplings of level-1 Higgs bosons with different SM and level-1 UED excitations [75]. To calculate the cross sections for different processes we make use of the CTEQ6L [76] parton distribution functions with the renormalization/factorization scale set at $\sqrt{\hat{s}}$. The present study is carried out at the parton-level to establish that cascade decays of level-1 KK particles of the MUED could turn out to be healthy sources for the (level-1) charged Higgs boson at the LHC that may aid their search.

\footnotetext{
${ }^{12}$ See, for example, Appendix A, equations A.3, A.4 and A.33 to A.36. of ref. 21].
} 


\subsection{Productions and decays: modes and rates}

Guided by the discussion above, in this subsection we analyze the production and decay schemes of different level-1 KK excitations that would have crucial bearing on the yield of $H_{1}^{ \pm}$at the LHC under UED cascades.

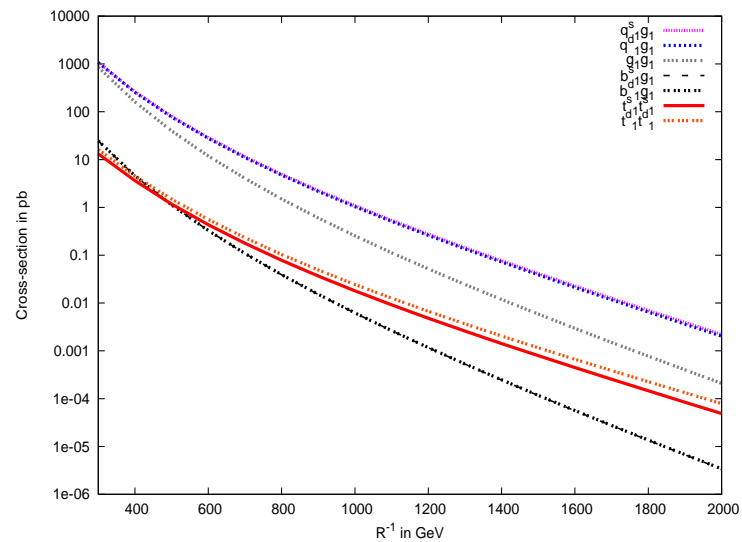

(a)

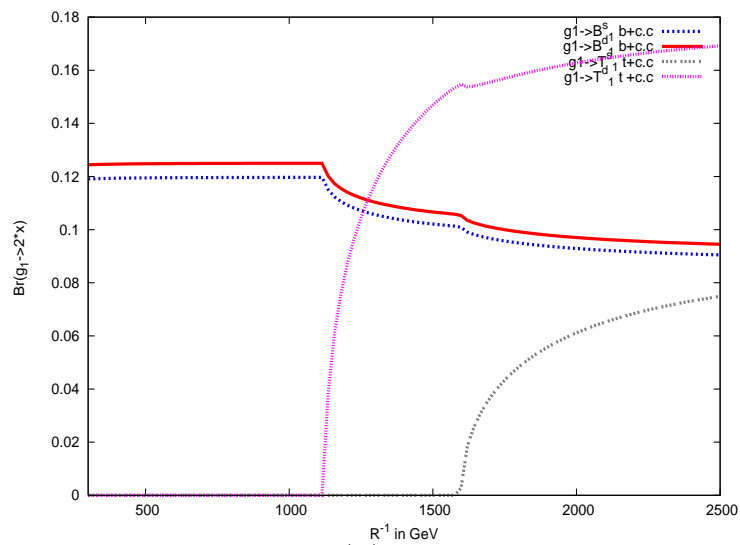

(b)

Figure 3: (a) Variations of different strong production cross sections at an LHC energy of $14 \mathrm{TeV}$. $q_{1}$ in the legend stands for the level-1 KK quarks from the first two generations. Contributions from the charge-conjugated final states are taken into account wherever relevant. (b) Variation of the decay branching fractions of level-1 KK-gluon to level-1 third generation KK-quarks as functions of $R^{-1}$.

In Fig. 3(a) we illustrate the basic strong production cross sections for $g_{1}$-pair, $g_{1} q_{1}$ (with first two generations of level-1 quarks) associated productions as a function of $R^{-1}$ at the LHC energy of $14 \mathrm{TeV} 13$ Also presented are the rates for the associated production of level-1 gluon along with level-1 bottom quarks and pair production of level-1 top quarks. We closely agree with the existing literature on these estimates at appropriate limits. From $g_{1}$-pair production and $g_{1} b_{1}$ associated production processes, a combinatoric enhancement in the rate for inclusive single $H_{1}^{ \pm}$final state would be present since both $g_{1}$ and $b_{1}$ can decay into $H_{1}^{ \pm}$. Pair productions cross sections of $b_{1}$ (including $b_{1}^{D} \bar{b}_{1}^{D}, b_{1}^{D} \bar{b}_{1}^{S}+$ c.c., $b_{1}^{D} b_{1}^{D}+$ c.c.) and $t_{1}^{S}$ are also indicated since they could directly decay into level-1 charged Higgs bosons. While obtaining the results presented in this plot we checked that the results of [4, 62] are reproduced under appropriate setups. In Fig. 3(b) we present the decay branching fractions of the level-1 KK gluon to the third generation level-1 quarks, i.e., $b_{1}$ and $t_{1}$, as functions of $R^{-1}$. In Figs. 4(a) and 4(b) similar variations of the subsequent decays of the level-1 lighter and heavier top quarks $\left(t_{1}^{S}\right.$ and $\left.t_{1}^{D}\right)$, respectively to $H_{1}^{ \pm}$are illustrated. A corresponding set of plots are presented in Figs 5 for the level-1 bottom quark, $b_{1}^{D}$.

\footnotetext{
${ }^{13}$ Later in this work, we demonstrate the prospect of a $10 \mathrm{TeV}$ LHC run which is imminent in a few years time after the scheduled take-off in November, 2009.
} 


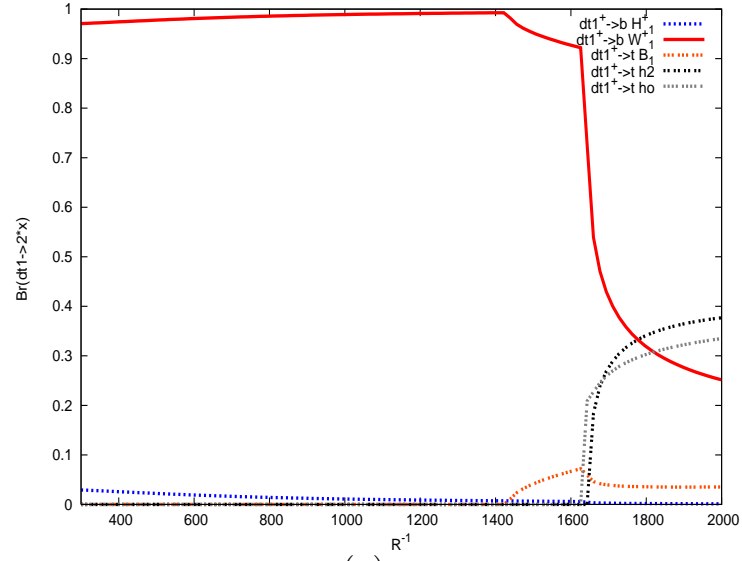

(a)

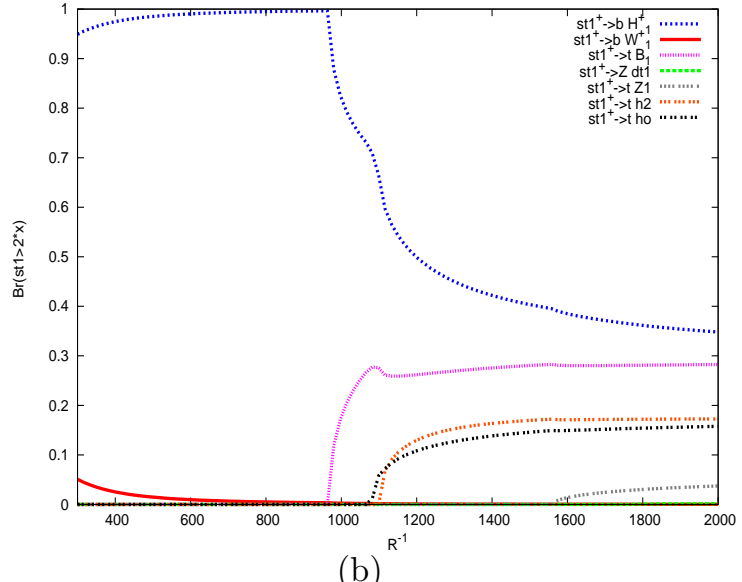

(b)

Figure 4: Variations of different decay branching fractions with respect to $R^{-1}$ (in $\mathrm{GeV}$ ) for (a) the lighter level-1 KK-top quark (predominantly doublet type) and (b) the heavier level-1 KK-top quark (predominantly singlet type).

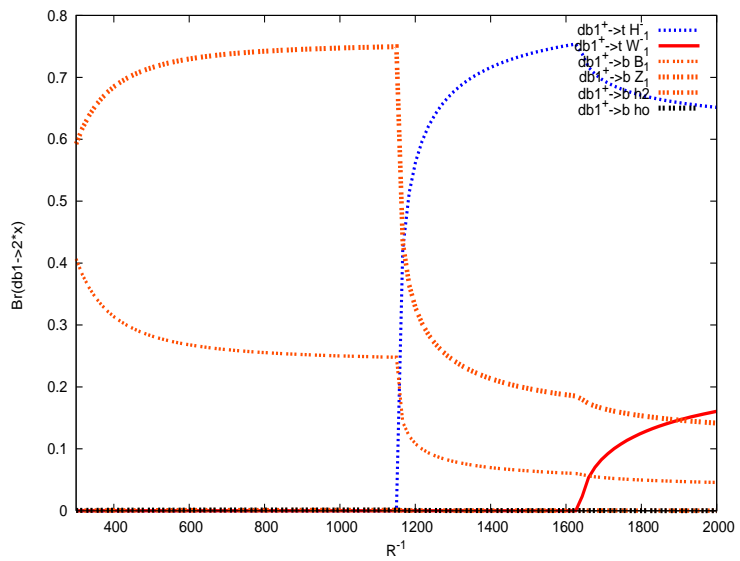

Figure 5: Variations of different decay branching fractions with respect to $R^{-1}$ (in $\mathrm{GeV}$ ) of the lighter (predominantly doublet type) level-1 KK-bottom quark. 
From Fig. 3(a) it is apparent that a simple sum over the cross sections from different production processes contributing to a final state with a single $H_{1}^{ \pm}$could vary between $1000 \mathrm{pb}$ and $10 \mathrm{fb}$ for $300<R^{-1}<2000 \mathrm{GeV}$. This is a conservative estimate since many of the contributing processes may have a combinatorial effect (as indicated above) which may lead to an enhanced effective cross section.

From Fig. 3(b) it is seen that $g_{1}$ could always decay into $b_{1}^{D}$ and $b_{1}^{S}$ (as far as third generation level-1 quarks are concerned, which may subsequently decay to $H_{1}^{ \pm}$). The decay $g_{1} \rightarrow t_{1}^{D} t$ opens up only at a higher value of $R^{-1}$ around $1100 \mathrm{GeV}$ when the mass-splitting between $g_{1}$ and $t_{1}^{D}$ allows for the top-mass. Once it opens up the corresponding branching fraction easily overtakes the ones for the decays to level-1 bottom quarks. The reason behind this is the appreciable mixing in the level-1 topquark sector compared to that in the level-1 bottom quark sector. As $m_{t_{1}^{S}}>m_{t_{1}^{D}}$, it is expected that $g_{1} \rightarrow t_{1}^{S} t$ opens up at an even larger value of $R^{-1}$. Note that, in any case, these branching fractions are only in the order of a few percent, the bulk fraction being shared by the decay modes to first two generations of quarks.

Figs. 4(a) and 4(b) reveal that it is only $t_{1}^{S}$ that can have an appreciable branching fraction to $H_{1}^{ \pm}$reaching almost $100 \%$ between $600<R^{-1}<950 \mathrm{GeV}$. Even for $R^{-1}$ around $2 \mathrm{TeV}$ the branching fraction to $H_{1}^{ \pm}$remains appreciable $(\simeq 35 \%)$. It is observed that the rest of the branching fraction is shared by $t_{1}^{S}$ decaying to level-1 neutral Higgs bosons or $B_{1}$ along with the SM top quark which are open for $R^{-1} \gtrsim 1 \mathrm{TeV}$. In other words, the small mass of the associated SM $b$-quark increases the possibility of an enhanced production of $H_{1}^{ \pm}$in the decay of $t_{1}^{S}$. Note that, the chiral mixing being not so large even for the level-1 quarks from the third generation, $t_{1}^{S}$ remains predominantly of the singlet type. Hence, its decay to $W_{1}^{ \pm}$is always suppressed. Just the reverse is true for $t_{1}^{D}$, i.e., it dominantly decays to $W_{1}^{ \pm}$before being taken over by decays to level-1 neutral Higgs bosons at around $R^{-1}=2 \mathrm{TeV}$. The decay patterns are consistent with the couplings involved whose forms are rather complicated (see, for example, Appendix A of ref.[21]).

Thus, as far as production of $H_{1}^{ \pm}$is concerned, the bottom line is that having $t_{1}^{D}$ would be of no use while $t_{1}^{S}$ could be a significant source (with a branching ratio $\gtrsim 35 \%$ ) over the phenomenologically interesting range of $R^{-1}$.

In Fig. 5 we present the decay branching fractions of $b_{1}^{D}$. Its decay to $H_{1}^{ \pm}$opens up for larger $R^{-1}(\simeq 1150 \mathrm{GeV})$ such that the mass-split between $b_{1}^{D}$ and $H_{1}^{ \pm}$allows for the mass of the accompanying top quark. It grows fast and dominates for large $R^{-1}$, the corresponding branching fraction reaching up to $65 \%$. The effects are again consistent with the form of the involved couplings mentioned above. On the other hand, $b_{1}^{S}$ decays into $b B_{1}$ with almost $100 \%$ branching fraction over the entire range of $R^{-1}$.

The summary of the information obtained from the above set of figures is the following. Only $t_{1}^{S}$ and $d_{1}^{B}$ can have appreciable branching fractions to $H_{1}^{ \pm}$with the 
former having the branching fraction to $H_{1}^{ \pm}$ranging between $35 \%$ and $100 \%$ over the accessible range of $R^{-1}$. However, having $t_{1}^{S}$ in the decay of $g_{1}$ is somewhat less probable and only takes place only at larger values of $R^{-1}$. On the other hand, $b_{1}^{D}$ can be obtained in the decay of $g_{1}$ (although with a branching fraction of only about $10 \%$, on an average, for $300 \mathrm{GeV}<R^{-1}<2000 \mathrm{GeV}$ ) this could yield $H_{1}^{ \pm}$under cascades for $R^{-1}>1150 \mathrm{GeV}$. Thus, it appears, that under cascade decays of gluino, $b_{1}$ induced $H_{1}^{ \pm}$production would exceed the one induced by $t_{1}$.

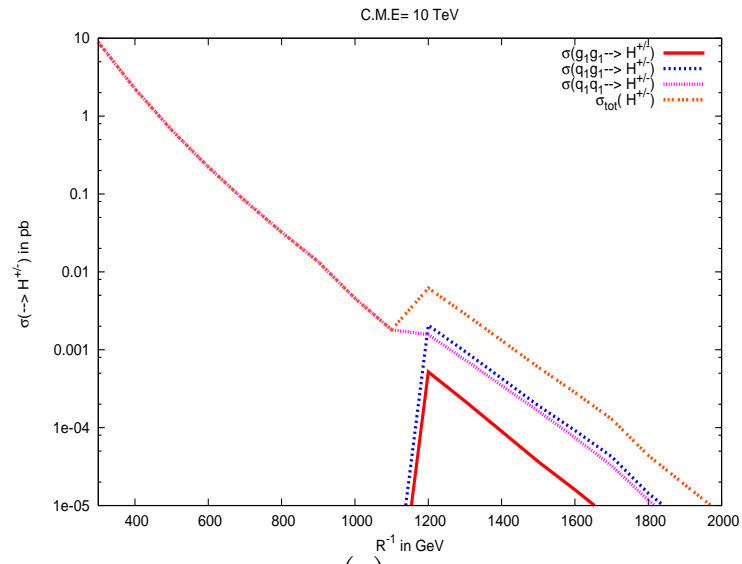

(a)

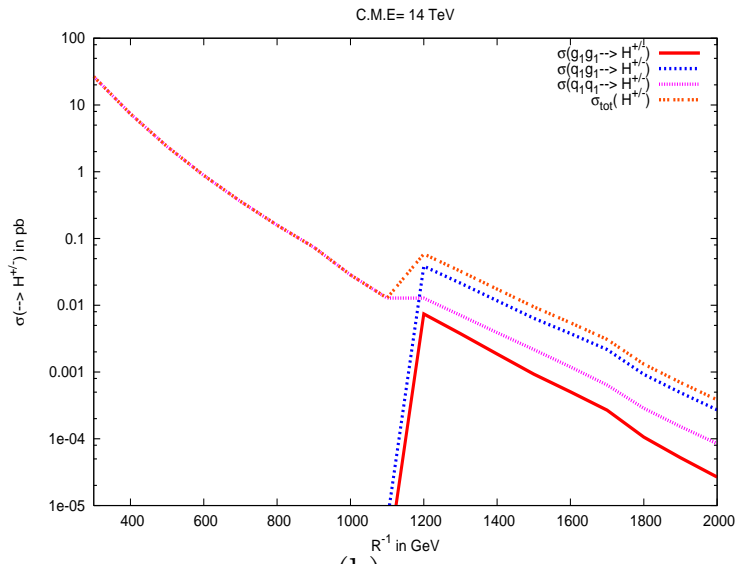

(b)

Figure 6: Variations of effective production cross sections of an inclusive KK level-1 charged Higgs bosons under cascade decays of UED excitations as functions of $R^{-1}$.

In Figs. 6(a) and 6(b) we compare the total contributions to $H_{1}^{ \pm}$production from these cascades and that from the direct productions of $t_{1}$ and $b_{1}$ at the LHC for centre of mass energies of 10 and $14 \mathrm{TeV}$ respectively. Up to about $1.1 \mathrm{TeV}$, the sole contribution to $H_{1}^{ \pm}$comes dominantly from the production of $t_{1}^{S} \bar{t}_{1}^{S}$-pair and their decays. As mentioned earlier, pair production of $b_{1}$ quarks could not play a role here since $H_{1}^{ \pm}$-s from their decays would always be accompanied by top quarks and the mass-split between $b_{1}$ and $H_{1}^{ \pm}$is not simply enough to accommodate the same for such low values of $R^{-1}$. As pointed out earlier, contribution to $H_{1}^{ \pm}$final states from the cascades of level-1 KK gluon turns on as soon as $R^{-1}$ is large enough $(\gtrsim 1.1 \mathrm{TeV})$ such that $g_{1} \rightarrow t_{1}^{S} \bar{t}$ opens up. There, as is clear from these figures, the contributions from the cascade decays could be appreciable and may enhance the yield of $H_{1}^{ \pm}$by an order of magnitude for both 10 and $14 \mathrm{TeV}$ runs. The observation is significant in view of not so big a rate for $H_{1}^{ \pm}$via direct productions of the level-1 $b$ and $t$ quarks. It has already been pointed out in earlier sections that detecting $H_{1}^{ \pm}$at the LHC could be a difficult task. This is because the decay products of $H_{1}^{ \pm}$can be rather soft and thus, an experiment like the LHC could turn out to be rather insensitive to them. Unless we may expect a radical improvement in the sensitivity of the experiment to softer decay products, the only way we could overcome this limitation is by having a larger rate for 
$H_{1}^{ \pm}$in the first place. This work advocates that the production of $H_{1}^{ \pm}$through UEDcascades could come into aid and may somewhat compensate for the poor sensitivity over a favourable range of $R^{-1}$. Interestingly enough, such an enhancement comes to our aid for heavier masses (higher values of $R^{-1}$ ) only thus could extend the reach of the LHC by 250-300 GeV (by looking at the effective cross sections) for both 10 and $14 \mathrm{TeV}$ runs. On the other hand, it can be seen from these figures that a 14 $\mathrm{TeV}$ run would match the yield from a $10 \mathrm{TeV}$ run with masses heavier by 300-400 $\mathrm{GeV}$. However, the absolute yields are predicted to be quite small for the $10 \mathrm{TeV}$ run. For example, requiring a minimum of 100 raw $H_{1}^{ \pm}$events before folding in the usual suppression factors like the lepton (particularly, $\tau$ ) reconstruction efficiency, triggerrelated factors and other kinematic cuts, the tentative reach in $R^{-1}$ at two different center-of-mass energies as a function of accumulated luminosities are as follows:

\begin{tabular}{||c||c|c|c|c||}
\hline \hline $\int \mathcal{L} d t\left(\mathrm{fb}^{-1}\right)$ & 1 & 10 & 50 & 100 \\
\hline \hline$\sqrt{s}=10 \mathrm{TeV}$ & 600 & 1000 & 1200 & 1400 \\
\hline$\sqrt{s}=14 \mathrm{TeV}$ & 900 & 1400 & 1500 & 1750 \\
\hline \hline
\end{tabular}

Table 1: Tentative reaches in $R^{-1}$ in $\mathrm{GeV}$ (by requiring 100 events at the level of effective production of $H_{1}^{ \pm}$from Figs. 6(a) and 6(b) as a function of accumulated luminosity for $10 \mathrm{TeV}$ and 14 $\mathrm{TeV}$ runs of the LHC.

This means that the $14 \mathrm{TeV}$ machine clearly has an edge over the $10 \mathrm{TeV}$ option as far as reach in $R^{-1}$ is concerned. However, as we can see from the Table 1, a moderate volume of data $\left(10 \mathrm{fb}^{-1}\right)$ could be what is needed at a $10 \mathrm{TeV}$ LHC run to probe $R^{-1} \sim 1 \mathrm{TeV}$. This is already encouraging.

It is also to be noted that reaching out to $R^{-1} \approx 1.4 \mathrm{TeV}$ cannot be a smooth exercise. For, as pointed out in section 3, with the SM Higgs mass set at $120 \mathrm{GeV}$, approaching $R^{-1} \approx 1.4 \mathrm{TeV}$ from the lower side would inevitably make $H_{1}^{ \pm}$the NLKP. This can make $H_{1}^{ \pm}$long-lived at the colliders and its signatures could be tantalizing enough.

\section{$5 \quad$ Summary and outlook}

We demonstrate that cascade decays of level-1 KK excitations of the MUED scenario could be a significant source of level-1 charged Higgs boson, $H_{1}^{ \pm}$, of the scenario. It is pointed out that cascades contribute to the yield of $H_{1}^{ \pm}$significantly only beyond a certain threshold value of $R^{-1}$ which is instrumental in generating the required masssplitting between the level-1 KK gluon $\left(g_{1}\right)$ and the level-1 top quark $\left(t_{1}\right)$ such that $g_{1}$ produced in hard scattering could decay into $t_{1}$ which in turn cascades down to $H_{1}^{ \pm}$. It 
is shown that with this added contribution to the yield of $H_{1}^{ \pm}$, the reach in $R^{-1}$ could be considerably enhanced, both at $10 \mathrm{TeV}$ and $14 \mathrm{TeV}$ runs with moderate amount of accumulated luminosity. However, some of the generic experimental challenges with possible signals of $H_{1}^{ \pm}$were also highlighted. A detailed generator-level analysis of the MUED Higgs sector including major detector effects is beyond the scope of the present work and can be discussed elsewhere.

The importance of detecting the level-1 MUED Higgs bosons should be put in perspective. It is clear that the spectra of level-1 UED Higgs bosons is similar to that of the Higgs bosons of the MSSM with the heavier Higgs bosons of the MUED being rather heavy $(\sim \mathcal{O}(\mathrm{TeV}))$ and degenerate (among themselves and also with other low-lying excitations of the MUED from the same KK-level). With the LHC running, a situation can be envisaged where one finds only one Higgs boson and no other resonances of a plausible new physics scenario (the so-called 'lone Higgs' scenario [66]) and one may like to identify this Higgs boson as that of the SM or the one from one of the scenarios beyond the same, like the MSSM, MUED or the Little Higgs scenario with conserved T-parity (LHT). It was demonstrated in ref. [66] that, for example, the size and the sign of deviation in the rate for $g g \rightarrow h \rightarrow \gamma \gamma$ from the SM expectation could be an indicator of the nature of the new physics. However, as long as the deviation at a given point of time remains small (say, below 30\%) it would not qualify to be a robust determinant, at least during the early stages of the experiment.

On the other hand, given the reach of the LHC can reasonably be expected to be in the same ballpark for both MSSM and the MUED, one could envisage an alternate possibility where one sees not only the Higgs boson but also some other MSSM-like excitations $(\sim \mathcal{O}(\mathrm{TeV}))$. However, it may turn out that the corresponding deviation from the SM expectation in the $g g \rightarrow h \rightarrow \gamma \gamma$ mode is not convincing enough for whatsoever reason. Such a situation could appear much confounding for two reasons: (i) the well-known SUSY-UED confusion for which an immediate identification of the observed resonances in favour of one or the other of these contending scenarios may not be possible, (ii) when the heavier Higgs bosons of the MSSM could still have showed up, in principle (since they could still be drastically lighter), even if the observed resonances (like the partners of strongly interacting quarks and gluon) are somewhat heavy (though observable), we are observing only one Higgs boson. This is not the case for MUED where the mass-scale of all the UED-excitations including the heavier Higgs bosons is determined by $R^{-1}$. An observation of this kind, i.e., non-observation of heavier Higgs bosons up to masses close to $R^{-1}$ that corresponds to the masses of the other observed resonances, may favour a scenario like the MUED, of course, without in any way, excluding the possibility of a SUSY like scenario, as such.

Under such circumstances, one may require to find, simultaneously, the corresponding neutral Higgs bosons of the emerging scenario in addition to the charged Higgs boson. For a given $R^{-1}$, their respective yields are expected to be somewhat smaller 
than the corresponding charged Higgs boson. On top of this, the neutral Higgs excitations being close in mass and being expected to be observed in a similar way, their individual resolutions are likely to get affected in any experimental setup. It is thus clear that nailing them down at the LHC would not be an easy task though could turn out to be a crucial one.

It may be reiterated that not only the Higgs sector of the MUED but also the whole spectrum of the scenario is rather degenerate at a given KK-level. Surely, the degeneracy is much severe and of a serious nature in the Higgs sector from the point of view of their detectability at a future collider. While the phenomenological implications of the MUED is worth studying in its own right (perhaps with a status similar to that enjoyed by the mSUGRA framework in SUSY studies, i.e., as a benchmark scenario with a somewhat tractable number of free parameters) its viability as a realistic framework should not be over-stressed. However, in the spirit of revealing and exploring diverse phenomenological possibilities at the future colliders in terms of novel and unexpected signatures and in the form of viable scenarios, such studies are very much called for and are being much advocated [72]. On the other hand, in recent times, studies revealed that even the framework of MUED could accommodate several alternate candidates

for the LKP, like the hypercharge gauge boson $B_{1}$, the charged Higgs boson $H_{1}^{ \pm}$and even the level-1 KK graviton $G_{1}$ [60] and thus potentially has the seeds that can lead to diverse phenomenological situations. There have also been attempts to obtain some robust, non-minimal realisations of the MUED [80] by including non-zero boundarylocalized terms at the cutoff scale $\Lambda$ for a part of the UED spectrum. Frameworks like these could enrich the resulting UED-phenomenology at future colliders, including that of the Higgs sectors of such scenarios.

\section{Acknowledgements}

The authors like to thank Bogdan Dobrescu, Monoranjan Guchait, Graham Kribs, Anirban Kundu, Andreas Nyffeler and Ashoke Sen for insightful discussions at different stages of the work. The work of BB has been supported by a research fellowship from the University Grants Commission, Government of India. BB thanks the Regional Centre for Accelerator-based Particle Physics (RECAPP), HRI (funded by the Department of Atomic Energy, Government of India under the XIth 5-year Plan) for a Young Associateship. AD thanks the Theory Division of CERN, Switzerland for their hospitality during the summer of 2009 when part of the present work was carried out. Computation required for this work are done on the computing facilities of RECAPP and the Cluster Computing Facility at HRI (http://cluster.hri.res.in). 


\section{References}

[1] T. Appelquist, H. C. Cheng and B. A. Dobrescu, Phys. Rev. D 64, 035002 (2001) arXiv:hep-ph/0012100].

[2] I. Antoniadis, Phys. Lett. B 246, 377 (1990).

[3] T. G. Rizzo, Phys. Rev. D 64, 095010 (2001) arXiv:hep-ph/0106336.

[4] C. Macesanu, C. D. McMullen and S. Nandi, Phys. Rev. D 66, 015009 (2002) [arXiv:hep-ph/0201300].

[5] H. C. Cheng, K. T. Matchev and M. Schmaltz, Phys. Rev. D 66, 036005 (2002) arXiv:hep-ph/0204342.

[6] H. C. Cheng, K. T. Matchev and M. Schmaltz, Phys. Rev. D 66, 056006 (2002) [arXiv:hep-ph/0205314].

[7] G. Bhattacharyya, P. Dey, A. Kundu and A. Raychaudhuri, Phys. Lett. B 628, 141 (2005) arXiv:hep-ph/0502031].

[8] M. Battaglia, A. Datta, A. De Roeck, K. Kong and K. T. Matchev, JHEP 0507, 033 (2005) arXiv:hep-ph/0502041.

[9] S. Riemann, In the Proceedings of 2005 International Linear Collider Workshop (LCWS 2005), Stanford, California, 18-22 Mar 2005, pp 0303 arXiv:hep-ph/0508136].

[10] B. Bhattacherjee and A. Kundu, Phys. Lett. B 627, 137 (2005) [arXiv:hep-ph/0508170].

[11] A. Datta, K. Kong and K. T. Matchev, Phys. Rev. D 72, 096006 (2005) [Erratumibid. D 72, 119901 (2005)] [arXiv:hep-ph/0509246].

[12] C. Macesanu, Int. J. Mod. Phys. A 21, 2259 (2006) arXiv:hep-ph/0510418.

[13] D. Hooper and S. Profumo, Phys. Rept. 453, 29 (2007) arXiv:hep-ph/0701197.

[14] B. Bhattacherjee, A. Kundu, S. K. Rai and S. Raychaudhuri, Phys. Rev. D 78, 115005 (2008) arXiv:0805.3619 [hep-ph]].

[15] S. Matsumoto, J. Sato, M. Senami and M. Yamanaka, arXiv:0903.3255 [hep-ph].

[16] G. Bhattacharyya, A. Datta, S. K. Majee and A. Raychaudhuri, Nucl. Phys. B 821, 48 (2009) arXiv:0904.0937 [hep-ph]].

[17] K. Agashe, N. G. Deshpande and G. H. Wu, Phys. Lett. B 514, 309 (2001) arXiv:hep-ph/0105084.

[18] K. Agashe, N. G. Deshpande and G. H. Wu, Phys. Lett. B 511, 85 (2001) arXiv:hep-ph/0103235].

[19] T. Appelquist and B. A. Dobrescu, Phys. Lett. B 516, 85 (2001) arXiv:hep-ph/0106140]. 
[20] D. Chakraverty, K. Huitu and A. Kundu, Phys. Lett. B 558, 173 (2003) arXiv:hep-ph/0212047.

[21] A. J. Buras, M. Spranger and A. Weiler, Nucl. Phys. B 660, 225 (2003) arXiv:hep-ph/0212143.

[22] J. F. Oliver, J. Papavassiliou and A. Santamaria, Phys. Rev. D 67, 056002 (2003) arXiv:hep-ph/0212391.

[23] A. J. Buras, A. Poschenrieder, M. Spranger and A. Weiler, Nucl. Phys. B 678, 455 (2004) arXiv:hep-ph/0306158.

[24] S. Khalil and R. Mohapatra, Nucl. Phys. B 695, 313 (2004) arXiv:hep-ph/0402225.

[25] P. Bucci and B. Grzadkowski, Phys. Rev. D 68, 124002 (2003) arXiv:hep-ph/0304121.

[26] P. Bucci, B. Grzadkowski, Z. Lalak and R. Matyszkiewicz, JHEP 0404, 067 (2004) arXiv:hep-ph/0403012.

[27] I. Gogoladze and C. Macesanu, Phys. Rev. D 74, 093012 (2006) arXiv:hep-ph/0605207.

[28] R. Mohanta and A. K. Giri, Phys. Rev. D 75, 035008 (2007) arXiv:hep-ph/0611068.

[29] K. R. Dienes, E. Dudas and T. Gherghetta, Nucl. Phys. B 537, 47 (1999) arXiv:hep-ph/9806292.

[30] H. C. Cheng, J. L. Feng and K. T. Matchev, Phys. Rev. Lett. 89, 211301 (2002) arXiv:hep-ph/0207125.

[31] G. Servant and T. M. P. Tait, Nucl. Phys. B 650, 391 (2003) arXiv:hep-ph/0206071].

[32] G. Servant and T. M. P. Tait, New J. Phys. 4, 99 (2002) arXiv:hep-ph/0209262.

[33] D. Majumdar, Phys. Rev. D 67, 095010 (2003) arXiv:hep-ph/0209277.

[34] D. Majumdar, Mod. Phys. Lett. A 18, 1705 (2003).

[35] M. Kakizaki, S. Matsumoto, Y. Sato and M. Senami, Phys. Rev. D 71, 123522 (2005) arXiv:hep-ph/0502059.

[36] M. Kakizaki, S. Matsumoto, Y. Sato and M. Senami, Nucl. Phys. B 735, 84 (2006) arXiv:hep-ph/0508283.

[37] F. Burnell and G. D. Kribs, Phys. Rev. D 73, 015001 (2006) arXiv:hep-ph/0509118.

[38] K. Kong and K. T. Matchev, JHEP 0601, 038 (2006) arXiv:hep-ph/0509119. 
[39] T. Flacke, D. Hooper and J. March-Russell, Phys. Rev. D 73, 095002 (2006) [Erratum-ibid. D 74, 019902 (2006)] arXiv:hep-ph/0509352.

[40] M. Kakizaki, S. Matsumoto and M. Senami, Phys. Rev. D 74, 023504 (2006) arXiv:hep-ph/0605280].

[41] J. M. Smillie and B. R. Webber, JHEP 0510, 069 (2005) arXiv:hep-ph/0507170.

[42] A. Datta, G. L. Kane and M. Toharia, arXiv:hep-ph/0510204.

[43] N. Arkani-Hamed, G. L. Kane, J. Thaler and L. T. Wang, JHEP 0608, 070 (2006) [arXiv:hep-ph/0512190].

[44] P. Meade and M. Reece, Phys. Rev. D 74, 015010 (2006) arXiv:hep-ph/0601124.

[45] C. Athanasiou, C. G. Lester, J. M. Smillie and B. R. Webber, JHEP 0608, 055 (2006) arXiv:hep-ph/0605286.

[46] L. T. Wang and I. Yavin, JHEP 0704, 032 (2007) arXiv:hep-ph/0605296.

[47] C. Athanasiou, C. G. Lester, J. M. Smillie and B. R. Webber, arXiv:hep-ph/0606212.

[48] D. Hooper and G. Zaharijas, Phys. Rev. D 75, $035010 \quad$ (2007) arXiv:hep-ph/0612137.

[49] S. Y. Choi, K. Hagiwara, H. U. Martyn, K. Mawatari and P. M. Zerwas, Eur. Phys. J. C 51, 753 (2007) arXiv:hep-ph/0612301.

[50] N. Arkani-Hamed, P. Schuster, N. Toro, J. Thaler, L. T. Wang, B. Knuteson and S. Mrenna, arXiv:hep-ph/0703088.

[51] L. T. Wang and I. Yavin, Int. J. Mod. Phys. A 23, 4647 (2008) arXiv:0802.2726 [hep-ph]].

[52] J. Hubisz, J. Lykken, M. Pierini and M. Spiropulu, Phys. Rev. D 78, 075008 (2008) arXiv:0805.2398 [hep-ph]].

[53] A. Belyaev et al., Pramana 72, 229 (2009) arXiv:0806.2838 [hep-ph]].

[54] M. Burns, K. Kong, K. T. Matchev and M. Park, JHEP 0810, 081 (2008) arXiv:0808.2472 [hep-ph]].

[55] G. Belanger, E. Nezri and A. Pukhov, Phys. Rev. D 79, 015008 (2009) arXiv:0810.1362 [hep-ph]].

[56] M. Burns, K. T. Matchev and M. Park, JHEP 0905, 094 (2009) arXiv:0903.4371 [hep-ph]].

[57] W. Ehrenfeld, A. Freitas, A. Landwehr and D. Wyler, JHEP 0907, 056 (2009) arXiv:0904.1293 [hep-ph]].

[58] T. Appelquist and H. U. Yee, Phys. Rev. D 67, 055002 (2003) arXiv:hep-ph/0211023. 
[59] U. Haisch and A. Weiler, Phys. Rev. D 76, 034014 (2007) arXiv:hep-ph/0703064.

[60] J. A. R. Cembranos, J. L. Feng and L. E. Strigari, Phys. Rev. D 75, 036004 (2007) arXiv:hep-ph/0612157.

[61] B. Bhattacherjee and A. Kundu, J. Phys. G 32, 2123 (2006) arXiv:hep-ph/0605118.

[62] B. Bhattacherjee and A. Kundu, Phys. Lett. B 653, 300 (2007) arXiv:0704.3340 [hep-ph]].

[63] F. J. Petriello, JHEP 0205, 003 (2002) arXiv:hep-ph/0204067].

[64] A. Datta and S. K. Rai, Int. J. Mod. Phys. A 23, 519 (2008) arXiv:hep-ph/0509277.

[65] S. K. Rai, Int. J. Mod. Phys. A 23, 823 (2008) arXiv:hep-ph/0510339.

[66] K. Hsieh and C. P. Yuan, Phys. Rev. D 78, 053006 (2008) arXiv:0806.2608 [hep$\mathrm{ph}]]$.

[67] See, for example, "Minimal Universal Extra Dimension in CalcHEP/CompHEP", A. Datta, K. Kong and K. T. Matchev, http://home.fnal.gov/ kckong/mued/.

[68] A. Pukhov, arXiv:hep-ph/0412191.

[69] M. ElKacimi, D. Goujdami, H. Przysiezniak and P. Skands, arXiv:0901.4087 [hepph].

[70] N. D. Christensen and C. Duhr, arXiv:0806.4194 [hep-ph].

[71] K. Kawagoe and M. M. Nojiri, Phys. Rev. D 74, 115011 (2006) arXiv:hep-ph/0606104.

[72] M. J. Strassler, arXiv:0811.1560 [hep-ph].

[73] A. Datta, A. Djouadi, M. Guchait and Y. Mambrini, Phys. Rev. D 65, 015007 (2002) arXiv:hep-ph/0107271.

[74] A. Datta, A. Djouadi, M. Guchait and F. Moortgat, Nucl. Phys. B 681, 31 (2004) arXiv:hep-ph/0303095.

[75] Private communications with Graham Kribs and Kyoungchul Kong.

[76] J. Pumplin, D. R. Stump, J. Huston, H. L. Lai, P. M. Nadolsky and W. K. Tung, JHEP 0207, 012 (2002) arXiv:hep-ph/0201195.

[77] R. Barate et al. [LEP Working Group for Higgs boson searches and ALEPH Collaboration and and], Phys. Lett. B 565, 61 (2003) arXiv:hep-ex/0306033.

[78] M. Guchait and D. P. Roy, arXiv:0808.0438 [hep-ph].

[79] G. L. Bayatian et al. [CMS Collaboration], J. Phys. G 34 (2007) 995.

[80] T. Flacke, A. Menon and D. J. Phalen, Phys. Rev. D 79, 056009 (2009) arXiv:0811.1598 [hep-ph]]. 